\title{
D01 Linking Frequent Seismic Time-lapse Surveys and Well Observations at the Valhall Field, Norway
}

\author{
Olav Barkved, BP
}

Seismic time-lapse surveys are increasingly being used to characterize the static and dynamic properties of our oil and gas reservoirs. The conventional approach is based on matching the seismic observations to predictions from simulator model through a history matching process. In 2003 we installed a permanent seismic array (LoFS) over the Valhall field, this allows for cost effective and highly repeatable time lapse surveys, to date 11 surveys have been acquired. In addition to the conventional use of matching seismic timelapse observations to the flow simulator state at certain stages we have had significant success in using the time-lapse observation directly in assessing individual well performance. The nature of the 4D seismic response and well completion design is such that we in many cases can see the performance of of individual perforations. Starting from qualitative evaluation we have developed automated procedures. The resulting "production profiles" has some similarities to how PLT data is presented and the notions Seismic PLT was introduced in cross-disciplinary discussions. "Projecting" the 4D response into a production profile has turned out to be a very useful basis for integrations across the subsurface and production disciplines.

The frequent surveying has also provided us with other intriguing results, like capturing the response during an injectivity test using. The higher pressure used in a short test differ from the normal injection test.

In the presentation I will give an overview of the Valhall LoFS array, summarize the 4D response but primarily discuss the use of the time-lapse data to support well production monitoring / optimization. In addition to discuss the specific injection test results, I will show some simple examples on insights related gained about the static model from the dynamic seismic time-lapse observations

And finally I will like to share some experiences related to terminology and using correct terms when moving outside our own specialized disciplines. 